\title{
The Fundamental Physics of Electromagnetic Waves
}

\author{
Juliana H. J. Mortenson \\ General Resonance, LLC \\ USA
}

\section{Introduction}

A new foundational physics is emerging which radically changes our concepts of electromagnetic waves. The original quantum ideas of Max Planck and Albert Einstein from the turn of the twentieth century, are undergoing an impressive renaissance now at the turn of the twenty-first century. The result is a fundamental physics of electromagnetic waves that is both new and classical. Einstein's insistence that quantum mechanics was incomplete - that "hidden variables" were yet to be discovered - was correct. The recent discovery of those variables is the driving force behind this rebirth of the foundations of quantum mechanics and the fundamental physics of electromagnetic ("EM") waves.

The new quantum variables have led to the discovery of new universal constants for EM waves. The new constants have revealed an elegant simplicity in quantum concepts, that requires no paradoxical explanations and imposes no uncertainties or limits. Instead, the new physics provides a more realistic understanding of physical concepts related to EM waves. The old paradigm is disappearing, and yielding to a new paradigm which is both more understandable and more powerful.

\section{Background}

It is often said that to successfully navigate the future one must understand the past. The fundamental physics of electromagnetic waves are no exception to this wisdom. In fact, an understanding of the origins of $20^{\text {th }}$ century physics regarding electromagnetic waves is of vital importance to understanding the scientific revolution that is currently taking place.

\subsection{Physics in the ages of reason and enlightenment}

Galileo Galilei (1564 - 1642) was one of the most influential scientists of the millennium, however he lived during a time when the protestant reformation was gaining momentum and Europe was in turmoil. The Catholic Church was losing its hold on much of northern Europe and the Thirty Years' War raged. Galileo resided on the Italian peninsula, where the Church maintained a strong hold, and he could not rely on the protection of reformers in other parts of Europe. None-the-less, even though "pagan" beliefs associated with frequency and resonancerelated phenomena had been banned by the Church for centuries, Galileo performed research on natural resonant frequencies in a pendulum system. (Mortenson, 2010b). 
In 1632, Galileo published his "Dialogue" and in a daring move described the mechanics of natural resonant frequencies writing, "the Pendulum makes its vibrations with one and the same frequency" and "every Pendulum hath the Time of its Vibrations...pre-fixed... [and] it is impossible to make it move under any other Period, than that ... which is natural unto it." (Galilei, 1632) He described the resonant accelerating forces produced by precisely time puffs of his breath stating, "by blowing upon [the Pendulum one may] confer a Motion, and a Motion considerably great by reiterating the blasts, but only under the Time properly belonging to its Vibrations". Galileo thus provided one of the first documented descriptions of resonance, namely the increase in amplitude and energy of a system's vibrations when an applied vibration, motion or energy matches the natural frequency of the system. Unfortunately, the Church was less accommodating than Galileo had anticipated. He was convicted of heresy and placed under house arrest for the rest of his life.

Pierre de Fermat (1601 - 1665) was a French attorney who was in his mid-thirties when Galileo was accused of heresy. Although Fermat's personal passion was mathematics, he was well aware that pursuit of certain mathematical subjects could be very dangerous. Thus Fermat engaged in his passion in secret, scribbling notes in the margins of books in his private library. One set of notes was a resonance equation, demonstrating that as the rate of a mechanical vibration (e.g., a puff of breath) neared the natural vibratory rate of a body (e.g., the swing of a pendulum), the amplitude of vibrations in the body increased (also see Figure 1., below):

$$
y=1 /\left(1+x^{2}\right)
$$

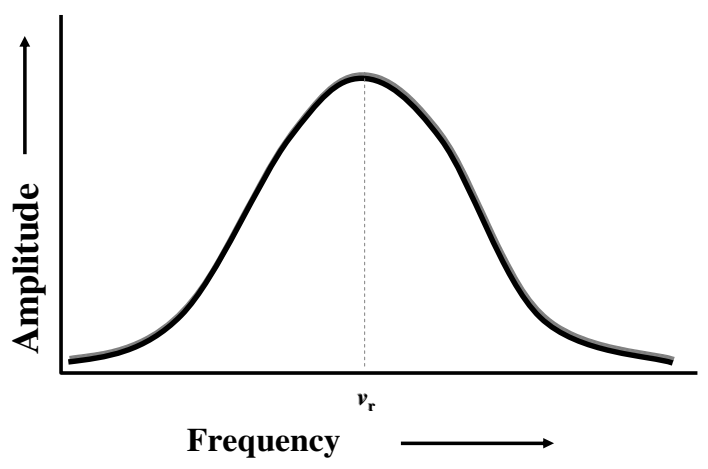

Fig. 1. Fermat's resonance curve showing an increase in vibration amplitude when forces are applied at natural resonant frequencies ( $\left.{ }^{\text {" }} v_{\mathrm{r}}{ }^{\prime}\right)$.

The brilliant young Isaac Newton $(1643$ - 1727) wrote his famous Principia, describing his three (3) laws of motion around the time of Fermat's death. (Newton, 1898) The religious climate in England was quite chaotic at the time, and Newton waited another twenty (20) years to actually publish his Principia. His second law (force equals mass times acceleration) provided the basis for yet another resonance equation:

$$
\mathrm{A}=\frac{\mathrm{a}}{\left|v_{r}{ }^{2}-v_{o}{ }^{2}\right|}
$$


where "A" is the amplitude of the system's oscillations, "a" is the acceleration in the system's oscillation (caused in Galileo's case by the force of his small puffs of breath), " $v_{r}$ " is the resonant or natural frequency of the system, and " $v_{\mathrm{o}}$ " is the frequency of the outside force applied to the system. As this second resonance equation shows, an outside force applied at a frequency which is either much higher or much lower than the natural resonant frequency of the system, produces a large denominator and hence a small amplitude. Conversely, the closer the frequency of the outside force is to the resonant natural frequency, the smaller the denominator becomes. Very large amplitudes are produced. When the outside frequency exactly matches the resonant frequency of the system the amplitude is theoretically infinite (Figure 2.).

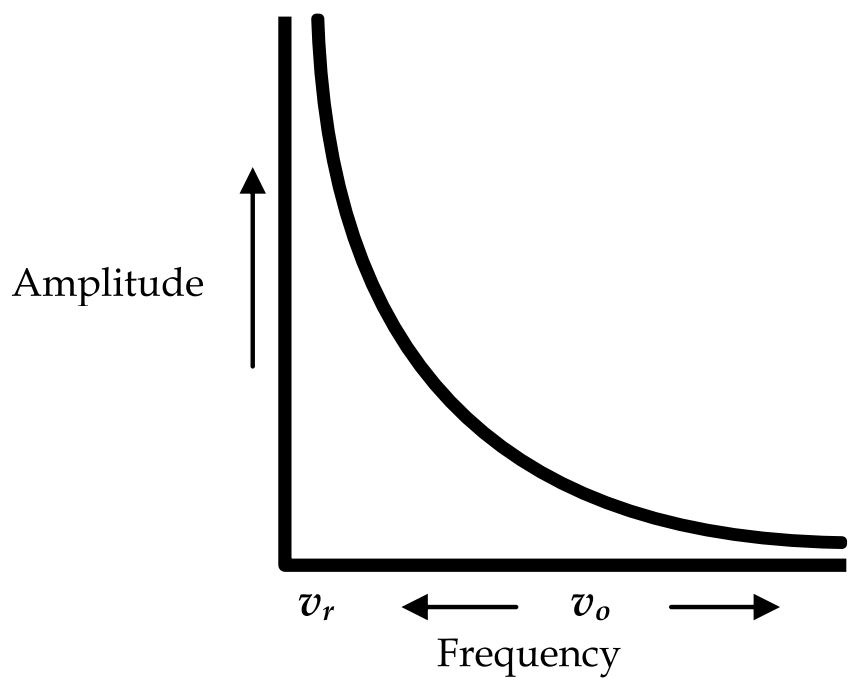

Fig. 2. Graphical representation of resonant amplitude equation (Eq. 2). The resonant frequency " $v_{r}$ " is at the origin, and input frequency of the outside force " $v_{o}$ " varies. As the input frequency approaches the resonant frequency, amplitude approaches infinity.

Newton distinguished the force exerted by an accelerating body, from the energy of a body simply in motion (which he referred to as vis viva) the product of mass and velocity:

$$
\text { vis viva }=\mathrm{m} \mathrm{v}
$$

where " $\mathrm{m}$ " is mass and " $\mathrm{v}$ " is velocity. This led to the great vis viva controversy several decades later (see below). By 1704 Newton had published his treatise "Opticks" in which he proposed the corpuscular theory of light, namely that light is composed of tiny particles that travel in straight lines. In a foreshadowing of Einstein's later work, Newton stated, "Are not gross Bodies and Light convertible into one another, ... and may not Bodies receive much of their Activity from the Particles of Light which enter their Composition?"

A few decades later the great vis viva controversy erupted with Giovanni Poleni's (16831761) proposal that vis viva energy was proportional to the product of mass and velocity squared, putting him at odds with Newton. The debate was soon joined by Leibnitz, Huygens, and others. Dutch physicist Willem Gravesande (1688 -1742) performed meticulous experiments and concluded that energy of motion, "follow[s] the Ratio compounded 
of the Masses, and the Squares of the Velocities" (underline added). (Gravesande, 1747) The noted French Newtonian scholar, Emilie du Châtelet (1706 - 1749) in her 1740 book, "Institutions Physiques" asserted that vis viva energy is proportional to the product of mass and velocity squared, based on Gravesand's painstaking experiments.

While the vis viva debate raged, the Italian mathematical prodigy Maria Gaetana Agnesi (1718-1799), published her 1748 book on calculus and differential equations, organizing the work of Fermat, Newton, Leibnitz and others. (Agnesi, 1748) She expanded on Fermat's resonance curve, providing a detailed geometric proof and a third resonance equation:

$$
y=h a^{2} / a^{2}+x^{2}
$$

where " $h$ " is the height of the curve and "a" the half-width at half-maximum. Her book was an immediate sensation throughout Europe, and resonance began to become a well known scientific principle, in spite of the English translation error that resulted in the resonance curve being known as the "Witch of Agnesi". (Spencer, 1940)

\subsection{Nineteenth century physics}

By the nineteenth century, the brilliant Joseph Louis Lagrange $(1736-1813)$ had organized the works of nearly every known scientist on matters of velocity, inertia, force, energy, and dynamics into his "Méchanique Analytique". (Lagrange, 1811) Lagrange declared that for a body at constant velocity, its energy (vis viva) was equal to " $\mathrm{mv}^{2}$ ", resulting "solely from the inertia forces of the bodies". Conversely, the energy required to accelerate a body was a function of the distance over which a force acted " $F \delta s$ ". Lagrange explained that all systems exhibited a dynamic equilibrium between the vis viva of constant velocity and the forces of acceleration, "The sum of these two quantities, when equated to zero, constitutes the general formula of dynamics... when the equilibrium does not hold, the bodies must necessarily move due to all or some of the forces which act on them." For purposes of systematically explaining analytic mechanics Lagrange stated that he had assumed that an acceleration always occurs in a time period at least as long as the unit time for velocity. His assumption effectively fixed the acceleration time interval at "one second" and excluded accelerations taking place in less than one second.

Lagrange also addressed resonance dynamics using a mathematical function: "in the case where the same function is a maximum, the equilibrium will not be stable and once disturbed the system will begin by performing fairly small oscillations but the amplitude of the [resonant] oscillation will continually grow larger." He included additional sections on "harmonics [at the] nodes of vibration", "the resonance of a sonorous body", and the resonance dynamics of pendulum oscillations.

Forty years later, Gaspard-Gustave de Coriolis (1792-1843) borrowed heavily from Lagrange's work in his popular engineering textbook. (Coriolis, 1829) Coriolis adopted Lagrange's assumption regarding the acceleration time interval for simplicity's sake, and explicitly explained that this assumption excluded consideration of "instantaneous" effects. Without the assumption, separate time variables for velocity and acceleration would have been required. Coriolis also introduced the concept of kinetic energy as a convenience in engineering applications involving gravitational effects: "the mass times one-half the square of the speed $\left[1 / 2 m v^{2}\right] \ldots$ will introduce more simplicity... since the factor ' $1 / 2\left(v^{2} / g\right)^{\prime}$ is nothing more than the height from which a heavy body... must fall so that it may acquire the speed ' $v$ '". Acutely aware that his kinetic energy formula did not apply to objects moving at constant velocity, Coriolis 
wrote that when "the speeds have become the same... [the kinetic energy] becom[es] zero". Coriolis' caveats were soon forgotten, however. By the time James Clerk Maxwell (18311879) later wrote his basic physics textbook, he errantly summarized, "The kinetic energy of a body is the energy it has in virtue of being in motion..."

Meanwhile, the interdisciplinary scientist Thomas Young, M.D., (1773 - 1829) began publishing physics articles anonymously (to protect the reputation of his medical practice). He eventually went public, and according to Young his greatest scientific achievement was establishment of the wave theory of light, based on his double slit experiment. Published exactly 100 years after Newton's Opticks, Young's reports on the wave-like interference of light eventually resulted in abandonment of Newton's light corpuscle theory. This led to development of the belief that matter was composed of small particles, and light composed of continuous waves.

Another interdisciplinary scientist - Hermann von Helmholtz, M.D., (1821 - 1894) - was an army surgeon who set up energy-related experiments on frogs in his army barracks. Those same biomechanical experiments led to his great treatise on the transformation and conservation of energy. (Helmholtz, 1889) Helmholtz's work on conservation of energy became the first law of thermodynamics, namely that energy is neither created nor destroyed, but is instead conserved and transformed from one form to another, "...heat, electricity, magnetism, light, and chemical affinity ... from each of these different manifestations of [energy] we can set every other [manifestation] in motion". Helmholtz carefully differentiated between orderly work energy and disorderly thermal energy, and taught that the total energy of a system was their sum:

$$
\mathrm{U}=\mathrm{A}+\mathrm{TS}
$$

where " $\mathrm{U}$ " is the internal energy of a system, " $\mathrm{A}$ " the work (Helmholtz) energy, " $\mathrm{T}$ " temperature, " $S$ " entropy, and the product " $T S$ " thermal energy.

Helmholtz also wrote extensively about resonance which is, "always found in those bodies which when once set in motion by any impulse, continue to perform a long series of vibrations before they come to rest ... provided the periodic time of the gentle blows is precisely the same as the periodic time of the body's own vibrations, very large and powerful oscillations may result. But if the periodic time of the regular blows is different from the periodic time of the oscillations, the resulting motion will be weak or quite insensible." (Helmholtz, 1862) He also described resonant coupling as "sympathetic resonance". Helmholtz eventually rose to the highest physics position in Germany at the University of Berlin, where he influenced many young students including Max Planck (1858 - 1947) and Heinrich Hertz (1857 - 1894). (Helmholtz, 1896 and 1904)

After Helmholtz challenged Hertz to prove the existence of Maxwell's theoretical EM waves, Hertz succeeded brilliantly. The new EM waves were called "resonant Hertzian waves", based on the resonant electrical processes Hertz used to transmit and receive them.

\subsection{The quantum revolution}

By the late 1800's, the young Max Planck was himself a professor at the University of Berlin and was doing theoretical work on Hertz's electromagnetic waves. (Planck 1896 and 1897) Planck modeled the EM waves on the one hand as resonant waves capable of producing orderly work energy " $\mathrm{A}$ ", and on the other hand as EM waves produced by random chaotic motions based solely on temperature "TS" (blackbody radiation). (Planck, 1900) Late in 1900 Planck met with success regarding the random thermal EM waves when he empirically 
determined the correct formula for blackbody radiation. A proper derivation of that empirical equation, however, was another matter altogether and according to Planck was the hardest work of his life. (Planck, 1901 and 1920)

Planck started with the Helmholtz equation $(\mathrm{U}=\mathrm{A}+\mathrm{TS})$ and then introduced his noncontroversial resonance hypothesis: EM "resonant Hertzian waves" are orderly and are thus completely free to be converted into work, and thereby constitute work energy, " $\mathrm{A}$ ". Planck next explained that, because the blackbody apparatus used in the laboratory had been specifically designed to exclude all resonant EM waves, he could assume there was no work energy in the blackbody device. According to Planck, "A" equaled zero, and thus "the entire problem is reduced to determining $S$ as a function of $U$ ". He borrowed Wilhelm Wien's method of solving for energy density (which eliminated a time variable) and also eventually resorted to the statistical methods of his arch nemesis, Ludwig Boltzmann (1844 - 1906).

Boltzmann's kinetic mechanics were based on the limiting assumption that all the elements (e.g., molecules or atoms) in a system were moving randomly, in a completely disordered manner. Boltzmann's mechanics were restricted to the thermal energy portion, "TS", of Helmholtz's energy equation and could not be applied to orderly work energy, " $A$ ". The blackbody device and experiments were deliberately designed to exclude work energy and measure only disorderly, chaotic thermal energy, however. This fact allowed Planck to use Boltzmann's statistical methods in his blackbody derivation, and "determin [e] S [solely] as a function of $U^{\prime \prime}$. It also required however, that Planck introduce his quantum hypothesis namely, that energy is quantized in small uniform amounts. Significantly, Planck assumed that those small uniform amounts of energy were different for each frequency, creating an $a$ priori limitation which excluded consideration of a unit of energy for EM waves, analogous to the unit of charge for electrons. Mathematically Planck's quantum hypothesis took the form of the quantum formula which Planck assumed as a given:

$$
\mathrm{E}=\mathrm{h} v
$$

where " $\mathrm{h}$ " is Planck's action constant, $6.626 \times 10^{-34}$ Joule seconds.

Planck also calculated a thermodynamic constant, now called the Boltzmann constant:

$$
\mathrm{E}=\mathrm{k}_{\mathrm{B}} \mathrm{T}
$$

where " $E^{\prime \prime}$ is the energy of a single element (e.g., a single atom or molecule) based solely on its temperature " $\mathrm{T}$ ", and " $\mathrm{k}_{\mathrm{B}}$ " is the Boltzmann constant, $1.38 \times 10^{-23}$ Joules per degree K per element. Just as Helmholtz's equation provided the energy of a macroscale system based on its temperature ("TS"), Planck's thermodynamic equation provided the energy of an individual microscale element based on its temperature (" $\left.\mathrm{k}_{\mathrm{B}} \mathrm{T}^{\prime \prime}\right)$. Thus, the Boltzmann constant is the microscale equivalent of entropy. Planck never introduced a microscale equivalent of the work energy " $\mathrm{A}$ ", however: the blackbody experiments excluded work energy. This resulted in a microscale energy formula which was necessarily incomplete for any system in which work energy was present.

While some scientists used Planck's blackbody equation for practical applications, his revolutionary quantum hypothesis received little attention - until, that is, Albert Einstein's (1879 - 1955) own revolutionary papers were published in 1905. (Einstein, 1905) Einstein had seized on Planck's quantum hypothesis and used it to provide explanations for a number of unexplained phenomena such as the photoelectric effect and ionization of gases. The interactions of EM waves and matter, he proposed, "appear more readily understood if one assumes that the energy of light is discontinuously distributed in space", e.g., in small particles or packets along the lines of Newton's "light corpuscles", and is absorbed in "complete units" or "quanta". 
Although highly controversial, Einstein's papers brought attention to Planck's quantum hypothesis and formula. A few years later, Niels Bohr (1885 - 1962) adopted Planck's quantum formula in his theory of the hydrogen atom. (Bohr, 1913) Controversy still raged however, and Robert Millikan (1858 - 1963) undertook a series of meticulous experiments testing the validity of Planck's constant and what he described as Einstein's "reckless" theories regarding energy quanta and photoelectric phenomena. (Millikan, 1916)

Millikan, well familiar with Planck's accepted resonance hypothesis distinguished the photoelectric effect as an ordered work function and not a thermal effect: "photoelectrons do not share in the energies of thermal agitation...absorption [of EM waves] is due to resonance (and we know of no other way in which to conceive it...)". Echoing Galileo, Millikan stated, "the phenomena of absorption and of emission show that...oscillators possess natural frequencies... and the characteristic waves which they emit are of these frequencies... if any particular frequency is incident upon such a substance the oscillators in it which are in tune with the impressed waves may be assumed to absorb the incident waves". Regarding the resonant work nature of the photoelectric effect he stated, "emission of [electrons] from the atom...takes place especially copiously when the impressed frequency coincides with a 'natural frequency'... [It] furnishes a proof which is quite independent of the facts of black-body [thermal] radiation, of the correctness of the fundamental assumption of the quantum theory, namely, the assumption of a discontinuous...energy absorbed by the electronic constituents of atoms from [EM] waves". (Underline added)

The quantum revolution begun by Planck and Einstein was taking hold.

\subsection{The quantum paradox}

As the quantum revolution began to gain momentum, paradoxes and puzzles began cropping up. The simple model of light waves and matter particles had been disrupted. Louis de Broglie (1892 - 1987) added to the confusion in the early 1920's when he proposed that if light could be both a wave and a particle, then so could matter. (de Broglie, 1924) Pursuing that line of reasoning, de Broglie found the lack of a unit of energy for EM waves, i.e., "an isolated quantity of energy" particularly troublesome. Without an energy constant for light (i.e., an isolated quantity of energy), de Broglie was unable to determine the fundamental mass of light using Einstein's energy-mass equivalence equation, " $\mathrm{E}=\mathrm{mc}^{2}$ ". Instead, the energy of light paradoxically depended on its frequency. De Broglie made the best of a conceptually difficult situation, and instead set Einstein's mass equivalence equation equal to Planck's quantum formula and solved for the rest mass of light at a particular frequency:

$$
\mathrm{m}_{0} c^{2}=\mathrm{h} v_{0} \text {, therefore } \mathrm{m}_{0}=\mathrm{h} v_{0} / c^{2}
$$

where " $\mathrm{m}_{0}$ " is the rest mass of light, and " $\mathrm{c}$ " the speed of light in vacuo. Since the number of different frequencies of EM waves are theoretically infinite, this approach produced a paradoxically infinite number of values for the rest mass of light. Unlike other particles such as the electron or proton, de Broglie could find no constant rest mass associated with light particles.

The lack of any energy or mass constants for light was quite puzzling indeed. Unbeknownst to de Broglie, Planck's limiting assumption about different quanta for each frequency excluded the very unit quantity of energy de Broglie sought. De Broglie could at least conclude however, that the rest mass of light in the visible region was quite small and in his Nobel prize speech explained, "The general formulae...may be applied to corpuscles of [visible] light on the assumption that here the rest mass $m_{0}$ is infinitely small... the upper limit of $m_{0} \ldots$ is approximately 10-24 gram. (de Broglie, 1929) 
De Broglie also used Planck's quantum formula to derive the momentum for light:

$$
\rho=\mathrm{m}_{0} \mathrm{c}=\mathrm{hv} / \mathrm{c}=\mathrm{h} / \lambda
$$

finding that the momentum of light appeared to be directly proportional to its frequency, and thus inversely proportional to its wavelength " $\lambda$ ". Once again, De Broglie obtained a zoo of values - this time for momentum since the range of frequencies and wavelengths in the EM spectrum is infinite.

In the meantime, Neils Bohr undertook his ambitious project modeling the hydrogen atom based on Planck's quantum formula and constant. Bohr found that he could not calculate time intervals in regard to the interactions between EM waves and electrons. He was forced to model instantaneously "jumping electrons" instead. (Bohr, 1913 and 1920) Few (including Bohr) were satisfied with the jumping electrons however, and in the mid-1920's two new approaches to quantum mechanics were introduced. In 1925, Werner Heisenberg, introduced matrix mechanics. (Heisenberg, 1925) A year later Erwin Schrödinger began publishing a series of papers on wave equations, intended to represent the real electron waves suggested by de Broglie. (Schrödinger, 1982)

Even with these two new approaches quantum mechanics still did not make sense to many early quantum pioneers. It lacked the certainty and definiteness of classical mechanics. Efforts to compensate for the many paradoxes included additional principles such as Heisenberg's uncertainty principle, and Bohr's complementarity principle. (Heisenberg, 1920 and Bohr, 1928) Additional variables and constants of inexplicable origin were discovered, such as the dimensionless fine structure constant. Discussion and debates continued. The Bohr-Heisenberg school of probabilities and uncertainty battled the Einstein - Schrödinger school of realism and certainty. Without answers for such simple matters as an energy constant or rest mass for light, the Bohr-Heisenberg school eventually prevailed. Scientists concluded (over Schrödinger's strenuous objections) that his wave equations represented only probabilities, and not real physical waves. The consensus that finally emerged was that the classical mechanics of our macroscale world simply could not be applied to the kaleidoscopic microscale world of the quantum. According to Bohr, a classical limit existed at the very highest electron energy levels in atoms, and below that limit classical mechanics simply could not be applied.

The iconoclastic brilliance which initially led Einstein to make his "reckless" quantum proposals, would not allow him to join the quantum crowd and he insisted that something had been missed. He simply could not believe that God and the universe were so perversely paradoxical. In 1935, Einstein published his "EPR" paper loudly proclaiming that quantum mechanics was incomplete due to the existence of "hidden" quantum variables. (Einstein, 1935) Einstein and others such as Bohm and Bell tried to describe the hidden variables, but such a task was difficult, if not impossible. (Bohm, 1952) How does one describe a quantum variable mathematically, when the very nature of the variable is unknown? Small groups of scientists have attempted to keep Einstein's quest alive, but the scientific community as a whole abandoned efforts to find any "hidden variables". Instead, it was generally agreed that the paradoxical nature of quantum mechanics was an undeniable reality of life. Incredible efforts then went into developing more quantum models incorporating the paradoxes, such as theories of strings, super-symmetry, membranes, and the like.

Einstein's stubborn insistence that something had been missed was correct, however. The first of his "hidden variables" was discovered nearly a century later, the result of a small 
mathematical thread. (Brooks, 2009,a) Tracing that thread through the historical record, it led to the discovery that a minor mathematical inadvertence in Planck's brilliant blackbody work had induced him to assume an incomplete and abbreviated version of the full quantum formula. All of quantum physics was based on Planck's simple quantum formula, and that assumed formula was incomplete: it was missing a time variable. After restoring the time variable, Planck's constant took on new fundamental meaning. The rich quantum tapestry that emerged, revealed beautifully symmetric quantum principles grounded in reality and certainty, using the complete quantum formula and a more inclusive or complete "thermo" dynamic formula. (Brooks, 2009,b)

\section{The complete quantum formula}

The complete quantum formula is:

$$
\mathrm{E}=\tilde{\mathrm{h}} \mathrm{t}_{\mathrm{m}} v
$$

where " $\tilde{\mathrm{h}}$ " is the energy constant for light $\left(6.626 \times 10^{-34}\right.$ Joules/oscillation) and " $\mathrm{t}_{\mathrm{m}}$ " is the measurement time variable.

\subsection{The time variable}

The complete quantum formula is quite similar to an energy relationship found in Planck's early theoretical electromagnetic work from the late 1890's. He converted time-based power measurements, " $\mathrm{E} / \mathrm{t}$ ", to total energy values by multiplying by the measurement time, " $\mathrm{t}_{\mathrm{m}}$ ". Planck's EM theory used that simple conversion in a generic relationship in which the oscillation energy of a system was proportional to the product of a generic constant "a", the measurement time variable, and frequency:

$$
\delta \mathrm{U} \approx \mathrm{a} \delta \mathrm{t}_{\mathrm{m}} v
$$

A few years later, the time variable was lost in Planck's complicated blackbody derivation. Instead of multiplying time-based energy measurements by the measurement time, Planck adopted Wien's mathematical methods which converted the power measurements into energy density values by dividing by the speed of light. This caused the measurement time variable " $\delta \mathrm{t}_{\mathrm{m}}$ " to be simultaneously fixed at a value of "one second", and then "hidden". Proof of these facts are found in Planck's 1901 blackbody paper, in which he described the experimental data and mathematical methods he used:

"§11. The values of both universal constants $h$ and $k$ may be calculated rather precisely with the aid of available measurement. F. Kurlbaum, designating the total energy radiating into air from $1 \mathrm{sq} \mathrm{cm}$ of a black body at temperature $t^{\circ} \mathrm{C}$ in $\mathbf{1} \mathbf{~ s e c}$, by $S_{t}$ found that:

$$
S_{100}-S_{0}=0.0731 \text { watt } / \mathrm{cm}^{2}=7.31 \times 105 \mathrm{erg} / \mathrm{cm}^{2} \underline{\mathrm{sec}}^{\prime \prime}
$$

Instead of multiplying Kurlbaum's time-based power measurement by the measurement time to obtain total energy (as Planck had done in his earlier work), he converted the power measurement to energy density by dividing by the speed of light " $c$ " $\left(3 \times 10^{10} \mathrm{~cm} / \mathrm{sec}\right)$, according to Wien's method:

"From this one can obtain the energy density of the total radiation energy in air at the absolute temperature 


$$
\frac{4 \cdot 7.31 \times 10^{5}}{3 \times 10^{10}\left(373^{4}-273^{4}\right)^{\prime \prime}}=7.031 \times 10^{-15} \mathrm{erg} / \mathrm{cm}^{3} \mathrm{deg}^{4}
$$

The time variables in the numerator and denominator cancelled out and Planck was seemingly able to address energy independent of time. Dividing by the constant speed of light however, is the same as multiplying by time:

$$
\frac{E / t s^{2}}{c}=\frac{E}{t s^{2}} x \frac{t}{s}=\frac{E}{s^{3}}
$$

where " $s$ " is distance. In this case the time value by which the power measurement was multiplied was the constant "one second" unit time of the constant speed of light. Planck seems to have been unaware that by using Wien's energy density calculation he was actually causing the infinitely variable measurement time to be fixed at a constant value of one second. He also seems to have been unaware that the fixed time variable was subsequently hidden in the final calculations of his action constant " $\mathrm{h}$ ":

$$
\mathrm{h}=6.626 \times 10^{-34} \text { Joule seconds }
$$

His action constant is actually the product of a true universal constant - " $\widetilde{\mathrm{h}}$ " - and the fixed, hidden measurement time variable, " $\mathrm{t}_{\mathrm{m}}$ ".

$$
\mathrm{h}=\widetilde{\mathrm{h}}_{\mathrm{m}} \text { where } t_{m}=1 \text { second }
$$

\subsection{The energy constant}

When the missing time variable is restored to the quantum formula, the identity of Planck's real universal constant becomes apparent. The hidden constant is, in fact, a universal energy constant, namely the energy of a single oscillation or EM wave. This universal energy constant for light is that same "isolated quantity of energy" de Broglie searched for, i.e., the fundamental small quantum of light's energy:

$$
\widetilde{\mathrm{h}}=6.626 \times 10^{-34} \text { Joules / oscillation }
$$

This fact is easily verified by solving Planck's incomplete formula for the energy of a single oscillation of light (see Brooks, 2009a for derivations). The numerical value Planck calculated for his action constant " $\mathrm{h}$ " is actually the numerical value of the mean oscillation energy of individual EM waves. The "isolated quantity of energy" hoped for by de Broglie, has been found.

The universal nature of this constant is made clear by consideration of the energy constant over a wide range of wavelengths, time periods and frequencies. The mean energy of a single EM wave remains constant regardless of whether it is a radio wave, microwave, infrared, visible or ultraviolet wave. For low frequency and long wavelength EM waves such as radio waves, the constant mean oscillation energy is spread out diffusely over a large volume of space. At higher frequencies and shorter wavelengths, the energy becomes more concentrated in a smaller volume of space. In the ultraviolet region, the energy of an oscillation becomes extremely dense, being confined to a very small region of space, around 100 nanometers or so in dimension. The amount of energy in a single oscillation is the same, however, regardless of the volume or time period it occupies. 
The constancy of the energy of a single EM wave over a variety of wavelengths and time periods means that the elementary quantum of light is constant over a shift in time or space. When a property is constant over a shift in time or space, that property is conserved and represents a universal property. The fundamental relationships are now clear. Just as the electron has a fundamental unit of charge which is conserved and represents a universal constant for electrons, light has a fundamental unit of energy, " $\tilde{\mathrm{h}}$ ", which is conserved and represents a universal constant for EM waves.

\subsection{The frequency variable}

Planck's quantum formula was incomplete, and as a result did not contain the oscillation energy constant. This in turn resulted in a quantum formula in which the units did not balance:

$\mathrm{E}($ Joules $)=\mathrm{h}($ Joule seconds $) v($ oscillations per second $)$, but Joules $\neq$ Joules oscillations

Scientists found they were unable to balance the quantum equations and use complete mathematical notation for frequency, namely cycles, waves or oscillations per second. As a result, mathematically incomplete notation, which omitted descriptive units for frequency's numerator, was adopted instead. Frequency is currently described in the International System of Units ("SI") as " $1 / \mathrm{sec}^{\prime}$ or "sec-1". This incomplete SI notation for frequency removes an essential mathematical element of reality in quantum mechanics.

Incomplete mathematical notation for frequency is no longer required to compensate for the deficiencies of the incomplete quantum formula. With the recognition of the energy constant - energy per oscillation - frequency can once again be correctly and completely notated as oscillations per unit time. The use of complete mathematical notation in quantum mechanics restores a vital aspect of mathematical reality. Recognition of "oscillations" in the numerator of frequency measurements provides a theoretical element corresponding to each element of reality in the complete quantum formula. As Einstein argued, such a correspondence is a critical requirement of a complete quantum mechanics.

\subsection{The photon}

In 1926, Gilbert Lewis coined the term "photon" for Einstein's light quantum. The energy of the photon was calculated with Planck's (incomplete) quantum formula, " $\mathrm{E}=\mathrm{h} v^{\prime}$. Questions have been raised from time to time since then, as to whether the "photon" is truly an indivisible particle of light. The answer to that question is now clearly, "No". The photon as previously defined is not an indivisible elementary particle.

The fixed time variable and energy constant had been hidden in Planck's "action" constant, and so it was not apparent to Lewis or others that what they were calling the "photon" was actually a time-based quantity of light energy, which relied on a fixed and arbitrary one second measurement time interval. A time-based amount of energy which relies on an arbitrarily defined time interval cannot be a fundamental or elementary particle of light. The photon is not an elementary particle of light.

What is the elementary particle of light, then? As identified by the universal energy constant, the elementary particle of light is the single oscillation of EM energy, i.e., a single cycle or wave of light. The elementary particle of light possesses the constant energy of $6.626 \times 10^{-34}$ Joules. It is the smallest known quantum of energy in the universe. What was 
labeled a "photon" by $20^{\text {th }}$ century physics is actually a collection or ensemble of these small elementary light particles. Each individual oscillation is a "complete unit" of light and can be emitted or absorbed as a complete and discreet unit.

The "photon" is not an indivisible particle of light, and is in fact a collection or ensemble of light oscillations, which can act separately and individually as complete energy units. Upon absorption by a detector or object, the energy of a collection of discreet oscillations can spread over several atoms or molecules, resulting in a multi-atom, energy distribution state known as "entanglement". (Brooks, 2009, c) This entanglement of EM energy can take place in different patterns or distributions, depending on the nature of the absorbing or detecting material. Similarly, emission of light energy can occur from an "entangled" energy state shared by multiple atoms or molecules in the emitter. An ensemble of EM waves with fewer than "N" oscillations (where " $v=\mathrm{N} / \mathrm{sec}^{\prime}$ ) results in a "sub-photonic" collection of EM waves. The ultimate sub-photonic particle is the elementary particle of light, the single EM oscillation.

\subsection{The mass of light}

De Broglie bemoaned the absence of "an isolated quantity of energy" with which he could calculate the constant rest mass of light. Using the energy constant for light, it is now possible to complete de Broglie's calculations and determine the rest mass of a single quantum of light. Under de Broglie's original formulation using Einstein's energy-mass equivalence equation of " $\mathrm{E}=\mathrm{mc}^{2}$ ", the rest mass of light is readily determined:

$$
\mathrm{m}_{0}=7.372 \times 10^{-51} \mathrm{~kg} / \text { oscillation }
$$

This value is within the same order of magnitude as the most recent and reliable estimates for the upper limits of the rest mass of light. Since the energy of a single oscillation of light is constant, regardless of its wavelength, time period or frequency, its mass is also constant regardless of its wavelength, time period or frequency. Hence, the mass of light is constant over a shift in time or space. The mass of light is thus conserved and represents another universal constant for light (Mortenson, 2011).

Just as the density of light's constant wave energy varies with the length and volume the wave occupies, the density of its mass varies as well. The mass of long EM radio waves, spread over a distance and volume of hundreds of meters, is low in density. The identical mass, when confined to the small wavelength and volume of an X-ray oscillation (on the order of $10^{-8}$ to $10^{-}$ 11 meters) is trillions of times more dense. High density X-ray oscillations, with their intensely concentrated mass and energy, can create interactions not typically seen with low density radio waves, and give rise to effects such as X-ray scattering and particle-like properties.

\subsection{The momentum of light}

Momentum is classically calculated as the product of an object's mass and its speed. Using the constant mass of an EM oscillation as calculated above, and the constant speed of light $\left(2.99 \times 10^{8} \mathrm{~m} / \mathrm{sec}\right)$, De Broglie's calculation for the momentum of light can be completed:

$$
\rho=\mathrm{m}_{0} \mathrm{c}=2.21 \times 10^{-42} \mathrm{~kg} \mathrm{~m} / \mathrm{sec} \text { per osc }
$$

As with mass, the momentum of a single oscillation of light is constant, rather than being infinitely variable. The momentum of an EM wave is constant regardless of its wavelength, 
time period or frequency. Thus the momentum of light is constant over a shift in time or space, and is a conserved property.

In terms of de Broglie's earlier calculations for the masses and momenta of photons, the mass and momentum constants for EM waves are not contradictory or confounding. It should be remembered that the photon of $20^{\text {th }}$ century concepts was actually a collection of elementary light particles, i.e., EM oscillations. Collections of masses and momenta can be additive. Summation of the constant mass and momentum of single oscillations (based on the number of oscillations " $\mathrm{N}$ " in a one second "photon") yields the same collective mass and momentum that de Broglie obtained with his photon-based calculations. Although de Broglie's mass and momentum calculations provided infinitely variable results, it is now recognized that his variable results were an artifact of the missing energy, mass and momentum constants. A previously unrecognized symmetry for conservation becomes apparent. Energy, mass and momentum are all conserved for both light and matter, completing the triad of conservation relationships outlined earlier by Helmholtz, Einstein and de Broglie.

\subsection{The force of light}

Energy, mass and momentum are all constant and conserved for light. Using classical mechanics, however, it is easily discerned that the force exerted by light is not constant. According to Lagrange, force is the product of mass and the change in velocity "during the instant $d t^{\prime \prime}$ when the velocity changes:

$$
\mathrm{F}=\mathrm{mv} / \mathrm{dt}
$$

For changes in velocity occurring in an interval of time equal to or greater then the velocity unit time, the same time variable for both velocity and acceleration can be used. If, on the other hand, the acceleration (or deceleration) occurs in a time interval much smaller than the velocity unit time (i.e., an "instantaneous" event), a second time variable, " $\mathrm{t}_{\mathrm{a}}$ ", must be used for the acceleration time interval. When an EM oscillation is emitted by an object, a small bit of mass of $7.372 \times 10^{-51} \mathrm{~kg}$ is instantaneously accelerated to the speed of light, " $c$ ". Likewise, when a light wave is absorbed by an object, its mass is instantaneously decelerated. The acceleration or deceleration occurs "during the instant $d t$ " which is the time period " $\tau$ " of the EM wave. The force that accelerates an EM oscillation at its emission (or that is exerted by an oscillation when it is absorbed) is thus:

$$
\mathrm{F}=\mathrm{mc} / \mathrm{t}_{\mathrm{a}} \text { where } \mathrm{t}_{\mathrm{a}}=\tau
$$

The time periods of EM waves are infinitely variable, as are their frequencies $(\tau=1 / v)$. Thus, although the mass and velocity of EM waves are constant, the forces which they exert are not. The forces associated with light oscillations vary inversely with their time periods, and directly with their frequencies (" $\mathrm{F}=\mathrm{m} \mathrm{c} v^{\prime \prime}$ ).

The energy and mass of a radio wave, distributed over a comparatively long period of time, exert relatively little force on an absorbing detector. The energy and mass of an X-ray or gamma ray oscillation, on the other hand, are concentrated in a minute period of time and exert tremendously large forces on an absorbing object.

These EM light forces are additive, and given sufficient accumulation the forces can be quite large and result in the physical acceleration of absorbing matter. (Liu et al, 2010) The force of light is the operative mechanism behind "space sails" which are now being employed on 
space craft. The sails of the ancient mariners were pushed by the forces of the wind which filled them. The sails of modern space explorers are now filled by the forces of light which impinge on them. Likewise, an object emitting light experiences a recoil force proportional to the emission force of the EM waves. (She et al, 2008)

\subsection{Classical limit}

The quantum pioneers anticipated that classical mechanics would be used to provide a description of physical processes at very small length and energy scales. Numerous roadblocks were encountered, however, due to the hidden quantum variables and constants. The quantum mechanics developed by Heisenberg and Schrödinger provided a mathematical framework for low energy kinetics, however they were unable to obtain the certainty and definitiveness provided by classical mechanics. Without the mass constant for EM waves, it was impossible to use classical properties of position, time, and mass in any meaningful way. Heisenberg and Bohr found that they were limited to finding just probabilities, and that they could apply classical mechanics only at very high electron energy levels. The region where the classical and quantum mechanics formed a boundary zone, was deemed the "classical limit" by Bohr. (Bohr, 1920) Above the limit, classical mechanics could be applied with reality and certainty, while below the limit all was uncertain and only quantum mechanics could be applied.

Using the new quantum variables and constants, the classical limit/boundary zone between quantum and classical mechanics is disappearing. (Mortenson, 2010,a) It is now possible to use classical mechanics at the smallest possible energy levels for light, equivalent to fractions of a percentage of the lowest known electron energy levels. The kinetics of energy absorption for a single EM oscillation, namely $6.626 \times 10^{-34}$ Joules, are now fully describable using classical mechanics. In this regard, the classical limit previously theorized by Bohr, is being recognized as an artifact of the missing quantum variables and constants.

The application of classical physics at the smallest known energy levels, is made possible with the use of the second hidden time variable, Lagrange's acceleration time variable, " $\mathrm{t}_{\mathrm{a}}$ ". The absorption or emission of an EM oscillation in the visible light region takes place in 10-10 seconds. This results in a near instantaneous deceleration or acceleration of light's mass. The energy required to accelerate a body is a function of the distance over which the force acts, " $F \delta s$ ". In the case of an individual EM oscillation, the distance over which the force acts is the wavelength, " $\lambda$ " of the oscillation. Multiplying the variable force for light by its wavelength, i.e., " $F \delta s=(\mathrm{m} \mathrm{c} v) \lambda$ ", results in constant energy of " $\mathrm{mc}^{2}$ ", or in other words $6.626 \times 10^{-34}$ Joules/osc. The energy constant for light is thus quickly derived from first principles of position, time and mass.

Lack of appreciation, for the caveats of Lagrange and Coriolis regarding acceleration time intervals and instantaneous events, contributed to the perception that a barrier or limit existed between classical and quantum mechanics. The new fundamental physics of EM waves reveals that particle mechanics can be described at both the macroscale and microscale levels using the certainty, realism and determinism of classical mechanics.

\subsection{The uncertainty principle}

Heisenberg suggested the uncertainty principle as a response to the inability of early quantum pioneers to determine quantum properties related to time or energy with any certainty. He proposed that changes in energy and time are uncertain to the extent that their 
product must always be greater than or equal to Planck's constant ( $\Delta \mathrm{E} \Delta \mathrm{t} \geq \mathrm{h}$ ). That principle included, of course, the incomplete quantum constant " $h$ ", which hid an energy constant and a fixed time variable. Heisenberg's uncertainty principle cured a multitude of quantum paradoxes, and as David Bohm wrote a generation later, "the physical interpretation of the quantum theory centers around the uncertainty principle". When " $h$ " is properly replaced with the energy constant and measurement time however, the physical interpretation of quantum theory is changed dramatically and centers around certainty and constancy, where the change in energy is the energy of a single EM wave, and the change in time " $\Delta t$ " and measurement time " $\mathrm{t}_{\mathrm{m}}$ " are equal to the time period " $\mathrm{t}$ " for the oscillation.:

$$
\Delta \mathrm{E} \Delta \mathrm{t} \geq \tilde{\mathrm{h}} \mathrm{t}_{\mathrm{m}} \text { and } \Delta \mathrm{E} \geq \tilde{\mathrm{h}}
$$

The smallest possible change in energy is the energy of a single wave of light.

This concept was obscured in the past due to the absence of a separate energy constant and time variable in Planck's quantum formula. Under the circumstances, it was inevitable that calculations of quantities involving time and energy, would yield uncertain results. The uncertainty is now gone, replaced by a quantum mechanics that accommodates a more certain and realistic physical interpretation.

\subsection{The fine structure constant}

The fine-structure constant "has been a mystery every since it was discovered more than fifty years ago, and all good theoretical physicists put this number up on their wall and worry about it....It's one of the greatest damn mysteries of physics: a magic number that comes to us with no understanding by man..." (Feinman, 1988)

Using the newly discovered quantum constants and variables, the fine structure constant " $\alpha$ " is far less of a mystery. Examination of the fine structure constant in relation to light's action "S" and Planck's constant " $h$ " (i.e., " $\alpha h=S$ "), and substitution of " $h$ " with the energy constant “ $\tilde{h}$ ” one finds:

$$
\alpha=\mathrm{S} \frac{1}{\tilde{\mathrm{h}}} \text {, or in other words } \alpha=(\mathrm{Et}) \frac{\mathrm{osc}}{\mathrm{E}} \text { and } \alpha=\text { osct }
$$

The fine structure constant is not dimensionless. It represents a scaling constant between time and a single oscillation of EM energy, i.e., "osc $t$ ". As such, a theoretical element corresponding to an element of reality is now provided for the fine structure constant. This is a critical requirement for a complete quantum mechanics.

\subsection{Wave - Particle duality}

Two opposing models of light - particles and waves - have been debated for centuries. Some investigations suggest light is composed of waves, while others suggest particles. This conundrum led Einstein to object, "“But what is light really? Is it a wave or a shower of photons? There seems no likelihood for forming a consistent description of the phenomena of light...we must use sometimes the one theory and sometimes the other...". (Einstein, 1938) Bohr responded to these two contradictory pictures of reality with his complementarity principle, asserting that certain aspects of light could be viewed one way or another, but never both at the same time.

We are now presented with a picture of reality which demands that we view light simultaneously as a wave and a particle. The elementary "particle" of light is the single EM 
oscillation or "wave". Although the time and space the wave occupies may vary, that variance is according to the constant ratio " $\mathrm{c}$ ", and the elementary particle's energy, mass and momentum remain constant as well.

The divergent pictures of the past resulted from relative size differences between the EM waves and the matter with which they interacted. For example, scattering studies were first performed using soft $\mathrm{X}$-rays with wavelengths larger than atoms, and no clear-cut particle properties were detected. When Arthur Compton used ultra-short hard X-rays and gamma rays, however (up to two orders of magnitude smaller than an atom) he observed particle-like properties. (Compton, 1923) The concentrated energy and mass of the X-ray and gamma ray waves appeared as small points relative to the size of the atoms in the irradiated materials.

On the other hand, one and two-slit experiments demonstrate wave-like properties for light via interference bands. These wave-like properties are also relative, however, to the sizes of the light oscillations and the matter with which they interact. For a slit whose width is equal to the wavelength of the light, no interference bands are observed and particle-like behavior is seen. It is only when the width of the slit is increased relative to the wavelength of the light that interference bands and wave-like properties begin to appear.

Recent experiments with light slits and "single photons" reveal as much about the detecting material as they do about the light itself. A "photon" is merely a collection of individual EM quanta. When visible light waves (which are much larger in size $(400-800 \mathrm{~nm})$ relative to the individual atoms in the detector $(0.1-0.5 \mathrm{~nm})$ ), strike a detector the energy of the light wave ensemble impinges on multiple detector atoms simultaneously. This produces an energy entanglement state in several of the detector atoms. (Brooks, 2009, c) Distribution of the light energy over several atoms excites a small point-like portion of the detector material resulting in a photonic reaction, and produces a particle-like pattern in the detector. (Roychouhuri, 2009) Although the resulting detector imaging appears to show the buildup over time of "photon" collisions, they actually show the buildup of energy entanglement states in the detector itself, which are subject to positive and negative interference within and between groups of entangled atoms.

\section{Energy dynamics}

The experimental data Planck used to derive the blackbody equation and thermodynamic formula did not include any measurements arising from orderly work energy. Hence, Planck did not include work energy in his thermodynamic formula, " $E=k_{B} T$ ". Instead, Planck's formulation was limited exclusively to the energy of a small system element (e.g., an atom, molecule or ion) based only on its temperature and random chaotic motion.

When orderly work energy is present in a system, more inclusive formulae must be used to represent the total energy of a system or its elements. (Brooks, 2009b and Mortenson, 2010b) Helmholtz's energy equation, " $\mathrm{E}=\mathrm{A}+\mathrm{TS}$ ", embodies once such inclusive formula on the macroscale, and represents the total energy of a system as the sum of its work and thermal energies. This more complete formula encompasses significantly more than simple thermodynamics, and is more appropriately referred to as an energy dynamics formula.

\subsection{Energy dynamics formula}

A complete energy dynamic formula for an entire system is given by Helmholtz's energy equation, "E = A + TS", (Equation 4., above). While calculation of the thermal energy of a system is relatively simple and straightforward, determination of the total work energy can 
be considerably more involved. Work energies come in many forms, including mechanical, chemical, gravitational and resonant energies. Resonance work energy is a broad category encompassing time-varying forces and fields such as sound waves, electric or magnetic fields, and light waves. These resonant energies couple to matter via "sympathetic resonance" and are denoted in the fundamental energy dynamics formula as, " $\mathrm{A}_{\mathrm{r}}$ ":

$$
\mathrm{E}=\mathrm{A}_{\mathrm{r}}+\mathrm{TS}
$$

The fundamental principle described by Galileo in his pendulum studies holds true for resonant work energies, i.e., "by [providing a time-varying energy one may] confer a Motion, and a Motion considerably great by reiterating...but only under the Time properly belonging to its Vibrations". Anyone who has pushed a child on a swing has applied a resonant mechanical energy to the child/swing system. Pushing the child at just the right time (i.e., the resonant frequency for the child/swing ensemble) increases the speed, height and excitement of the child's ride. Pushing at the wrong time, when the child is a few meters away, produces no effect on the system and may detract from the excitement of the child's ride.

In the same way, electromagnetic waves impinging on a material transfer resonant EM energy to the absorbing matter via their momentum, force, speed and mass. An acceleration of the oscillating element within the system results from the applied EM force, and an increase in the oscillation amplitude of that element results (see Fig. 1, above). Thus, "pushing" the system elements with EM waves at just the right time increases the amplitude (height) of the system's oscillations and excites them to higher energy levels. The amount the system's oscillation amplitude increases is a function of how close the resonant EM wave frequency is to the oscillation frequency inside the system (Eq. 2., and Fig. 2, above).

The increased oscillation amplitudes and energy levels in the system can perform work in a variety of ways, depending on which element or oscillation amplitude is increased. For example, changes in motion, chemical, material, organizational, or behavioral states may all result from a resonant energy excitement in the system.

Expressed at the microscale level, a complete energy dynamics formula for the total energy of an individual element in a system is formulated parallel to Helmholtz's system formula:

$$
\mathrm{E}=\mathrm{W}_{\mathrm{e}}+\mathrm{k}_{\mathrm{B}} \mathrm{T}
$$

where " $\mathrm{W}_{\mathrm{e}}$ ", is the total microscale work variable representing the total work performed on an individual element. In the case of resonance work energy, a resonance work variable, " $\mathrm{r}_{\mathrm{A}}$ " can be used. This microscale resonance work variable represents the energy gained by an individual element in a system, as a result of resonance work energy, " $\mathrm{A}_{\mathrm{r}}$ ", applied to the system as a whole:

$$
\mathrm{E}=\mathrm{r}_{\mathrm{A}}+\mathrm{k}_{\mathrm{B}} \mathrm{T}
$$

\subsection{Determination of system resonance work energy "Ar"}

The resonance work energy (system/macroscale) and variable (element/microscale) may be determined experimentally. An aqueous solvent system under resonant conditions was compared to an identical system under thermal conditions (see Table 1., below): ${ }^{1}$

${ }^{1}$ Experimental Procedure - Distilled water $\left(500 \mathrm{ml}\right.$ at $\left.20^{\circ} \mathrm{C}\right)$ was placed in each of two 1,000 ml beakers. One beaker was irradiated with resonant vibrational electromagnetic frequencies of water for three (3) hours, by a light 


\begin{tabular}{|l|l|l|}
\hline & Resonant system & Thermal system \\
\hline Weight Dissolved $(\mathrm{g} / 100 \mathrm{ml} \mathrm{NaCl})$ & 26.0 & 23.8 \\
\hline Moles Dissolved $(\mathrm{NaCl})$ & 4.65 & 4.25 \\
\hline Heat of solution $(\mathrm{kJ})$ & 17.4 & 16.0 \\
\hline
\end{tabular}

Table 1. Resonant vs. Thermal aqueous solvent system

The heat of solution is a measurement of the work performed by the solvent on the dissolving solute. The work performed by the resonant system was $17.4 \mathrm{~kJ}$, while the thermal system performed only $16.0 \mathrm{~kJ}$ of work on the $\mathrm{NaCl}$ solute. The energy dynamics formulae for both systems are:

$$
\begin{aligned}
\text { Thermal system } \mathrm{E}_{\mathrm{T}}=\mathrm{TS} \text {, and } 16.0 \mathrm{~kJ} & =\left(274^{\circ} \mathrm{K}\right) \mathrm{S} \\
\text { Resonant system } \mathrm{E}_{\mathrm{R}}=\mathrm{A}_{\mathrm{r}}+\mathrm{TS} \text { and } 17.4 \mathrm{~kJ} & =\mathrm{A}_{\mathrm{r}}+\left(274^{\circ} \mathrm{K}\right) \mathrm{S}
\end{aligned}
$$

Subtracting, one finds that the resonance work energy, " $\mathrm{A}_{\mathrm{r}}$ ", in the resonant system is $1.4 \mathrm{~kJ}$ of energy:

$$
\mathrm{A}_{\mathrm{r}}=1.4 \mathrm{~kJ}
$$

\subsection{The resonance factor}

The ratio of the total energy in the resonant system to the total energy in the thermal system:

$$
\mathrm{E}_{\mathrm{R}} / \mathrm{E}_{\mathrm{T}}=\mathrm{r}_{\mathrm{f}}
$$

is the resonance factor, " $\mathrm{r}_{\mathrm{f}}$ ". In the aqueous solvent system described above, the resonance factor is 1.09 . There was $9 \%$ more energy available in the resonant system to perform work on the solute and to dissolve it. This resonance work energy was in addition to the thermal energy already inherent in the system as a result of its temperature.

\subsection{Determination of element resonance work energy " $r_{A}$ "}

The amount of resonance work energy at the microscale is the resonance work variable, " $\mathrm{r}_{\mathrm{A}}$ ". In the solvent system example, individual elements in the system irradiated with resonant EM waves possessed greater energy than the elements in the thermal system. The value of the microscale resonance work energy can be calculated using Equation 25., above:

source using $2.1 \mathrm{~kJ}$ total energy. The other beaker was placed in an opaque incubator for three (3) hours. The water in both beakers at the end of the three (3) hours was $23^{\circ} \mathrm{C}$. Sodium chloride $(250 \mathrm{~g})$ was added to each beaker, and the beakers were stored identically in a darkened cabinet. Twenty (20) hours later temperatures of the solutions were identical $\left(21^{\circ} \mathrm{C} / 274^{\circ} \mathrm{K}\right)$. The solutions were decanted and the dissolved weight, salinity and concentration measurements of the resonant and thermal saline solutions were made using standard methods. (Brooks et al, 2005) 


$$
\begin{gathered}
\text { Thermal element } E_{t}=k_{B} T \text { and } E_{T}=405 \times 10^{-23} \text { J per molecule } \\
\text { Resonant element } E_{r}=r_{A}+k_{B} T \text { and } E_{r}=440 \times 10^{-23} \text { J per molecule }
\end{gathered}
$$

Subtraction shows that each water molecule in the resonant system performed an additional $35 \times 10^{-23} \mathrm{~J}$ of work on the solute, as a result of absorption of the resonant EM waves.

\subsection{Virtual thermal effects of resonant EM waves}

When resonant EM waves perform work on a system and increase one or more oscillation amplitudes within the system, that increased oscillation energy is free to be transformed into work within the system. In the case of the aqueous solvent system described in the experimental example above, the vibrational oscillations of the solvent water molecules were excited. This in turn led to a change in the behavior of the water as a solvent. The resonant water dissolved $26.0 \mathrm{~g} / 100 \mathrm{ml}$ of $\mathrm{NaCl}$, while the thermal water dissolved only $23.8 \mathrm{~g} / 100 \mathrm{ml}$.

To what temperature would the thermal system need to be raised, in order to dissolve the same amount of $\mathrm{NaCl}$ that the resonant system dissolved, all else being equal? This is readily calculated by setting the total element energy in the resonant system equal to the thermal energy, and solving for temperature " $\mathrm{T}$ ":

$$
\begin{gathered}
\mathrm{E}_{\mathrm{r}}=\mathrm{k}_{\mathrm{B}} \mathrm{T} \text {, therefore } \mathrm{T}=\mathrm{E}_{\mathrm{r}} / \mathrm{k}_{\mathrm{B}} \\
\mathrm{T}=319^{\circ} \mathrm{K}\left(46^{\circ} \mathrm{C}\right)
\end{gathered}
$$

In order to dissolve the same amount of $\mathrm{NaCl}$ in the thermal system, that the resonant system had dissolved, the thermal system would have had to be heated to $46^{\circ} \mathrm{C}$. The water in the resonant system behaved as though it had been heated to $46^{\circ} \mathrm{C}$, even though it had not. The EM waves provided a virtual thermal effect in the resonant solvent system.

\subsection{Energy efficiency of resonant EM waves}

As Helmholtz described many decades ago, energy can be transformed and converted from one form to another, "...heat, electricity, magnetism, light, and chemical affinity ". The efficiencies with which these transformations take place is not uniform across all conversions of energy. Depending on the process and desired end-result or product, the energy transformation efficiency can vary widely. For example, in the water solvent example given above, one would need to heat the water to $46^{\circ} \mathrm{C}$, in order to dissolve the same amount of salt that the resonant water system had dissolved. Heating $500 \mathrm{ml}$ of water to that higher temperature would require at least $52 \mathrm{~kJ}$ of energy.

On the other hand, the light source which provided the resonant vibrational EM waves to the resonant system consumed only $2.1 \mathrm{~kJ}$ of energy. (Mortenson \& George, 2011). The total additional energy required to achieve the desired end-result or product (i.e., dissolve more salt), is far less with the resonant EM waves: ninety-six percent (96\%) less, in fact.

The total extra heat of dissolution work performed by the resonant water on the solute was $1.4 \mathrm{~kJ}$. The energy transformation efficiency for the resonant system was $67 \%$. If one were to heat the thermal water to increase its temperature by $25^{\circ}$, using the $52 \mathrm{~kJ}$ of energy, the efficiency of the thermal energy conversion into heat of dissolution would be only $3 \%$. There is more than an order of magnitude difference between the energy conversion efficiency of the resonant EM system and the thermal system. 


\subsection{Boltzmann weighting}

Under Boltzmann mechanics for purely random and chaotic thermal systems, the elements (e.g., molecules) in a system adhere to a thermal distribution curve (Figure 3.a., below). In general terms, at low temperatures, most of the elements or molecules are at the lowest possible energy level or ground state. As the temperature in the system increases, the elements begin to leave the lower energy levels and populate the upper energy levels. At very high temperatures, several of the upper energy levels may be populated, leaving few molecules in the lowest ground state. The distribution of elements in the energy levels is determined with the Boltzmann factor:

$$
e^{-E_{n} / k_{B} T}
$$

This is a weighting factor that determines the probability that an element will be in the " $\mathrm{n}^{\text {th }}$ " energy state when the system is in thermodynamic equilibrium. The Boltzmann factor excludes consideration of resonant work energies (which are orderly), and assumes completely random motions in the system. Helmholtz energies are thus typically assumed to be at a minimum when Boltzmann mechanics are applied.

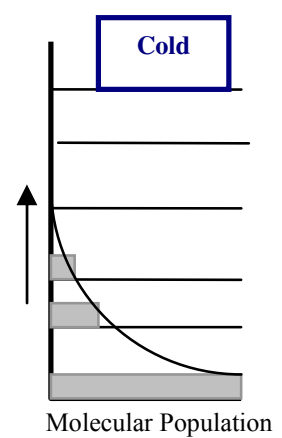

a. Boltzmann thermal distribution
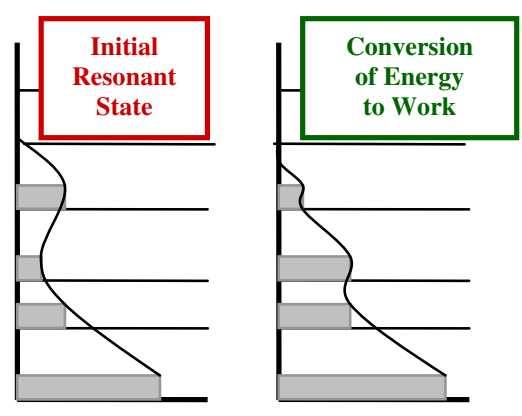

b. Resonant distribution

(Non-Boltzmann)

Fig. 3. Comparison of energy level population states under thermal conditions and resonant EM conditions. Upper energy level populations are increased as temperature increases. Absorption of resonant EM waves produces an irregular resonant energy distribution curve. This can result in system behavior equivalent to a "virtual" thermal distribution curve.

When a system is exposed to resonant EM waves, a "virtual" thermal effect can be produced, as in the aqueous solvent example above. In such a case, the "virtual" thermal distribution may be determined using a modification to the Boltzmann weighting factor:

$$
e^{-\mathrm{E}_{\mathrm{n}} / \mathrm{r}_{\mathrm{f}} \mathrm{k}_{\mathrm{B}} \mathrm{T}}
$$

in which the resonance factor, " $\mathrm{r}_{\mathrm{f}}$ " is included. The resulting thermal distribution curve reveals the energy state distribution curve of the thermodynamic system that would result in the same desired product or behavior that is produced by the system absorbing resonant energy.

The assumptions of randomness in the Boltzmann mechanics do not apply to resonant EM systems, with their uniform work energies, and systems exposed to resonant EM waves do 
not follow the smooth thermal distributions curves, however. Individual energy levels in the system may be selectively populated, changing the shape of the traditional smooth curve, to a bulging or "lumpy" energy distribution curve. (Figure 3b., above). Absorption of resonant EM waves initially results in increased population of an upper energy level. As the energy is converted to work in the system, the energy state devolves and relaxes. When all of the work energy has been spent, a thermal distribution is once again exhibited. Depending on which energy level(s) are selectively populated, the work performed will vary and can include speeding the rate of a reaction in a catalytic manner, e.g., virtual thermal effects can replace chemical activation energies. (Fukushima J. et al, 2010)

\subsection{Equilibrium constant}

In chemical and materials systems the work performed by the resonant EM waves can also shift the equilibrium of the system and produce dramatic changes in its chemical and material dynamics. In the dynamic equilibrium of chemical and material systems, in which reactants are transformed into products at the same rate products are transformed back into reactants, the equilibrium constant " $\mathrm{K}$ " indicates the point of dynamic equilibrium (productto-reactant concentration ratio). Systems with a large equilibrium constant contain mostly product, while a low " $\mathrm{K}$ " indicates mainly reactants.

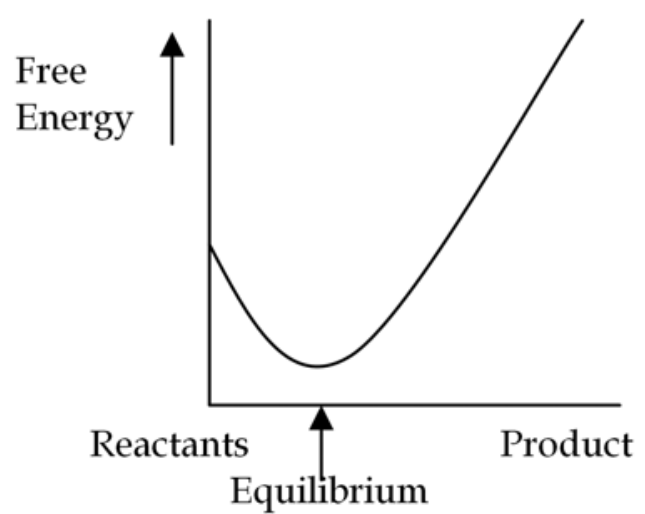

Fig. 4. a. Thermal system

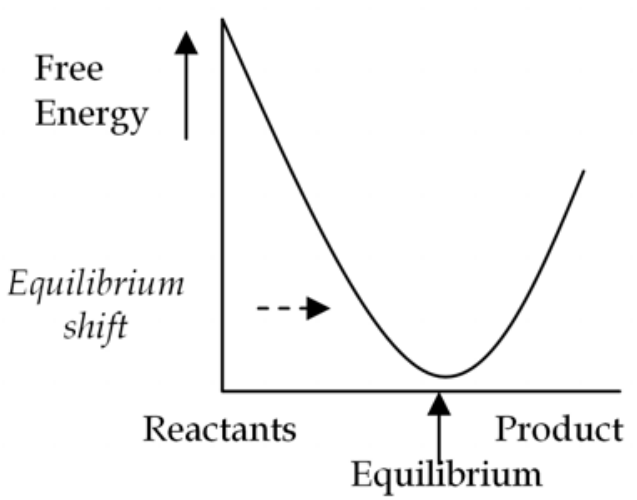

Fig. 4. b. Resonant system

In statistical thermodynamics the equilibrium constant is proportional to another natural $\log , \mathrm{K}$ $\approx \mathrm{e}^{-} \Delta \mathrm{E} / \mathrm{RT}^{\prime \prime}$ (where $\Delta \mathrm{E}$ is chemical free energy). When resonant $\mathrm{EM}$ waves are present in a system, the resonance factor properly appears in the denominator of the power notation, i.e., " $\mathrm{r}_{\mathrm{f}} \mathrm{RT}$ " to reflect the total increase in system energy. If the resonant EM waves increase chemical free energy, " $\Delta \mathrm{E}^{\prime}$, the ratio of chemical free energy to system energy remains the same. The equilibrium constant does not change, however the EM waves act as a catalyst and increase the rate of the reaction. (If the chemical free energy decreases, i.e., the resonance factor is less than "one", the EM waves will act as a negative catalyst and slow the rate of reaction.)

When resonant EM waves are absorbed and transformed to something other than chemical free energy, then $\Delta \mathrm{E} / \mathrm{r}_{\mathrm{f}} \mathrm{RT}<\Delta \mathrm{E} / \mathrm{RT}$, and the equilibrium constant will increase. Resonant EM waves that perform useful work on a system can thus increase the equilibrium constant, " $\mathrm{K}$ ", and increase the actual concentration of desired products. Resonant EM waves can 
cause a shift of the equilibrium curve. (Figure 4.b., above) In other words, resonant EM waves can achieve results not obtainable with classical catalysis or thermodynamics. (Brooks and Abel, 2007, Blum et al, 2003, Fukushima H., 2010, and Roy et al, 2002)

\section{Conclusion}

The two fundamental formulae which formed the foundations of quantum mechanics and $20^{\text {th }}$ century physics were both incomplete in regard to electromagnetic waves. The quantum formula was missing a time variable and energy constant, due to a minor inadvertence in Max Planck's derivation of the blackbody equation. Unforeseen consequences occurred as a result. The identity of the true elementary particle of light - the single EM oscillation - was obscured. Mathematical nomenclature for frequency became incomplete. Calculations of the mass, momentum, and force of EM waves were made impossible. Paradoxical principles including the classical limit, the uncertainty principle, and the complementarity principle were made necessary. Dimensionality of the fine structure constant was hidden, and great confusion arose over the wave vs. particle nature of light. Use of the complete quantum formula remedies these difficulties and provides a sound foundation for a certain and realistic quantum mechanics.

Likewise, the thermodynamic formula derived in Planck's blackbody work was not an inclusive or complete formula for energy dynamics. The complete energy dynamics formulae allow the resonant EM work energies in systems to be accounted for mathematically, both at the macroscale and microscale. Traditional Boltzmann mechanics cannot be strictly applied to EM waves, because Boltzmann mechanics assume completely random motions. Absorption of uniform EM waves requires modifications of Boltzmann weighting. Resonant EM waves can provide virtual thermal effects, decreased energy requirements, and increased energy efficiencies. Depending on how their energy is converted to work, resonant EM waves can act as catalysts - changing chemical or materials reaction rates - or they can shift reaction equilibria altogether, producing effects and products not seen or obtainable under typical thermodynamic conditions.

A new and powerful scientific paradigm is being revealed in the fundamental physics of electromagnetic waves.

\section{References}

Agnesi, M. G. (1748). Instituzioni Analitiche, Nella Regia-Ducal Corte, Milan, Italy

Blum, B.; Brooks (now Mortenson), J.; \& Mortenson, M. (2003). Methods for controlling crystal growth, crystallization, structures and phases in materials and systems, U.S. Patent Application No. 10/508,462, 2003.

Bohm, D., (1952). A Suggested Interpretation of the Quantum Theory in Terms of "Hidden" Variables, Physical Review, Vol.85, No.2, pp. 166-179

Bohr, N., (1913). On the Constitution of Atoms and Molecules, Part I, Philosophical Magazine Vol.26, pp. 1-24

Bohr, N. , (1920). Uber die Serienspektra der Element, Zeitschrift für Physik, Vol.2, No.5, pp. 423- 478, English translation in Niels Bohr Collected Works, Vol. 3: The Correspondence Principle (1918-1923), (1976). Nielsen, (Ed.), Amsterdam: NorthHolland Publ., pp. 241-282 
Bohr, N., (1928). The Quantum Postulate and Atomic Theory, Nature, Vol.121, No.3050, pp. 580-590

Compton, A., (1923). Quantum Theory of the Scattering of X-rays by Light Elements, The Physical Review, Vol.21, No.5, pp. 483-502

Brooks (now Mortenson), J.; Mortenson M.; \& Blum, B. (2005). Controlling chemical reactions by spectral chemistry and spectral conditioning. U.S. Patent Appl. No. 10/507,660, 2005.

Brooks (now Mortenson) J. \& A. Abel (2007). Methods for using resonant acoustic and/or resonant acousto-EM energy, U.S. Patent No. 7,165,451

Brooks (now Mortenson), J. (2009). Hidden Variables: The Elementary Quantum of Light', Proc. of SPIE Optics and Photonics: The Nature of Light: What are Photons? III, Vol.7421, pp. 74210T-3, San Diego, California, USA, August 2009

Brooks (now Mortenson), J. (2009). Hidden Variables: The Resonance Factor, Proc. of SPIE Optics and Photonics:, The Nature of Light: What are Photons? III, Vol.7421, pp. 74210C, San Diego

Brooks (now Mortenson), J. (2009). Is indivisible single photon essential for quantum communications...? No. 4, Proc. of SPIE, The Nature of Light III, 2009, Vol. 7421, pp. 74210Y, San Diego, California, USA, August 2009

Coriolis, G. (1829). Calculation of the Effect of Machines, Carilian-Goeury, Paris, France

De Broglie, L. (1924). Researches on the quantum theory, Thesis, Paris, France

De Broglie, L. (1929). The Wave Nature of the Electron, Nobel Lecture, Stockholm, Sweden, 1929

Einstein, A. (1905). On a Heuristic Point of View Concerning the Production and Transformation of Light, Annalen der Physik, Vol.17, pp. 132-148

Einstein,A.; Podolsky, B.; \& Rosen, N. (1935). Can a Quantum-Mechanical Description of Physical Reality be Considered Complete?, Physical Review, Vol.47, pp. 777-780

Einstein, A. \& Infeld, L. (1938). The Evolution of Physics, Simon and Schuster Inc., New York, New York, USA pp. 262-263

Feinman, R. (1988). QED. The Strange Theory of Light and Matter. Princeton Univ. Press, Princeton,

New Jersey, USA, pp. 129

Fukushima, H. (2010). New saccharification process of cellulosic biomass by microwave irradiation, Proc. of Mat. Sci. E Tech. 2010, Pittsburg, PA, USA, Oct. 2010, pp. 2859 2863

Fukushima, J. , Takayama, S., Matsubara, A., Kashimura, K. and Sato, M. (2010). Reduction Behavior of $\mathrm{TiO}_{2}$ During Microwave Heating, Proc. of MSET 2010, Pittsburg PA, pp. $2831-2836$

Galilei, G. (1632). Dialogue Concerning the Two Chief World Systems, English translation available at:

http://archimedes.mpiwg-berlin.mpg.de/cgi-bin/toc/toc.cgi? step=thumb\&dir =galil_syste_065_en_1661

Gravesande, W. J. (1747). Mathematical Elements of Natural Philosophy Confirm'd by Experiments, Third Edition, Book II, Chapter 3, Innys, Longman and Shewell, London, pp. 193-205

Heisenberg, W. (1925). Über quantentheoretische Umdeutung kinematischer und mechanischer Beziehungen, Zeitschrift für Physik, Vol.33, pp. 879-893

Heisenberg, W. (1927). Über den anschaulichen Inhalt der quantentheoretischen Kinematik und Mechanik. Zeitschrift für Physik, Vol.43, pp. 172-198

Helmholtz, H. (1862). On the Sensations of Tone, Longmans, Green, and Co., New York, NY 
Helmholtz, H. (1889). Ostwald's Kalssiker der Exacten wissenschaften, Über die Erhaltung der Kraft. Verlag von Wilhem Engelmann, Leipzig, Germany

Helmholtz, H. (1986). The Correlation and Conservation of Forces: A Series of Expositions, D. Appleton and Co., New York, NY, USA, pp. 211-250

Helmholtz, H. (1904). Popular Lectures on Scientific Subjects, Lecture VI. On the Conservation of Force, Trans. Atkinson E., Longmans, Green and Co., New York, NY, USA, pp. 306

Lagrange, J. L. (1811). Analytical Mechanics, Trans. Boissonnade A. \& Vagliente, VN, 1997, Kluwer Academic Publishers,Norwell, MA, USA

Liu, M. , Zentgraf, T. , Liu Y. and Bartal G., Light-driven nanoscale plasmonic motors, Nature Nanotechnology, Published online: July 4, 2010 | DOI: 10.1038/NNANO.2010.128

Millikan, R. A. (1916). A Direct Photoelectric Determination of Planck's "h", Phys Rev, Vol.7, No.3

Mortenson (formerly Brooks), J (2010a). Double Your Kin. Energy: Disappearance of the Classical Limit in Quantized Classical Energy Mechanics. Proc. MSET 2010, pp. 2907-2918, 2010a.

Mortenson (formerly Brooks) , J (2010b). The Fall and Rise of Resonance Science. Proceedings of MSET 2010, pp. 2865 - 2875, Houston, TX, USA, October 2010

Mortenson (formerly Brooks), J and George, R. (2011). The New Physics and Global Sustainability. IEEExplore, 978-1-4244-9216-9/11/IEEE FSNC, Vol.2, Earth Sci. \& Eng., pp. 332-335

Mortenson (formerly Brooks), J (2011). The Conservation of Light's Energy, Mass and Momentum, Proc. Of SPIE Optics and Photonics 2011, In press

Newton, I. (1887). Philosophiae Naturalis Principia Mathematica, Royal Society, London

Planck, M. (1896). Absorption und Emission electrischer Wellen durch Resonanz, Ann. der Phys. und Chem., Vol.293, No.1, pp. 1-14

Planck, M. (1897). Ueber electrische Schwingungen welche durch Resonanz erregt und durch Strahlung gedämpft warden", Ann. der Phys. und Chem., Vol.296, No.4, pp. 577-599

Planck, M. (1900). Ueber irreversible Strahlungsvorgänge, Ann.Phys., Vol.306, No.1, p 69-122

Planck, M. (1901). On the Law of Distribution of Energy in the Normal Spectrum", Annalen der Physik, Vol.309, No.3 pp. 553-63

Planck, M. (1920). The Genesis and Present State of Development of the Quantum Theory, Nobel Lectures, Physics 1901-1921, Elsevier Publishing Company, Amsterdam

Roy, R., et al. (2002). Definitive experimental evidence for Microwave Effects", Materials Research Innovation, Vol.6, pp. 128-140

Roychouhduri, C. (2009), Exploring divisibility and summability of 'photon' wave packets in non-linear optical phenomena, Proc. of SPIE, The Nature of Light III, 2009, Vol. 7421, p 74210A.

Schrödinger, E. (1982). Collected Papers on Wave Mechanics, Quantization as a Problem of Proper Values (Part I), Chelsea Publ. Co., New York, NY, USA, pp. 1-12.

She, W.; Yu, J.; \& Feng, R.(2008). Observation of a Push Force on the End Face of a Nanometer Silica Filament Exerted by Outgoing Light, PhysReview Letters, Vol.101, No. 24

Spencer, R. C. (1940). Properties of the Witch of Agnesi - Application to Fitting the Shapes of Spectral Lines, Journal of the Optical Society of America, Vol.30, pp. 415 - 419 


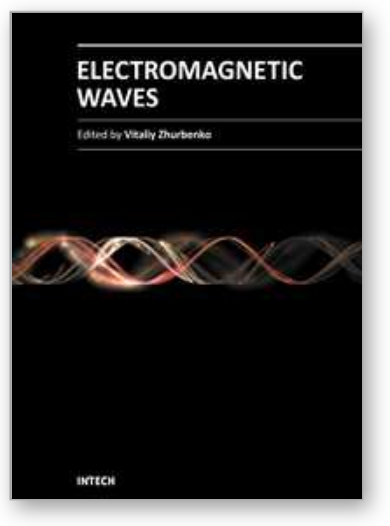

\author{
Electromagnetic Waves \\ Edited by Prof. Vitaliy Zhurbenko
}

ISBN 978-953-307-304-0

Hard cover, 510 pages

Publisher InTech

Published online 21, June, 2011

Published in print edition June, 2011

This book is dedicated to various aspects of electromagnetic wave theory and its applications in science and technology. The covered topics include the fundamental physics of electromagnetic waves, theory of electromagnetic wave propagation and scattering, methods of computational analysis, material characterization, electromagnetic properties of plasma, analysis and applications of periodic structures and waveguide components, and finally, the biological effects and medical applications of electromagnetic fields.

\title{
How to reference
}

In order to correctly reference this scholarly work, feel free to copy and paste the following:

Juliana H. J. Mortenson (2011). The Fundamental Physics of Electromagnetic Waves, Electromagnetic Waves, Prof. Vitaliy Zhurbenko (Ed.), ISBN: 978-953-307-304-0, InTech, Available from:

http://www.intechopen.com/books/electromagnetic-waves/the-fundamental-physics-of-electromagnetic-waves

\section{INTECH}

open science | open minds

\author{
InTech Europe \\ University Campus STeP Ri \\ Slavka Krautzeka 83/A \\ 51000 Rijeka, Croatia \\ Phone: +385 (51) 770447 \\ Fax: +385 (51) 686166 \\ www.intechopen.com
}

\author{
InTech China \\ Unit 405, Office Block, Hotel Equatorial Shanghai \\ No.65, Yan An Road (West), Shanghai, 200040, China \\ 中国上海市延安西路65号上海国际贵都大饭店办公楼 405 单元 \\ Phone: +86-21-62489820 \\ Fax: $+86-21-62489821$
}


(C) 2011 The Author(s). Licensee IntechOpen. This chapter is distributed under the terms of the Creative Commons Attribution-NonCommercialShareAlike-3.0 License, which permits use, distribution and reproduction for non-commercial purposes, provided the original is properly cited and derivative works building on this content are distributed under the same license. 